clearly influences parental response, as was shown by the differences reported for parents having infants born at different

\title{
Infants in a neonatal intensive care unit: parental response
}

\section{Redshaw}

\section{A commentary on the article by Carter et al (see page 109)}

T he admission of a baby to neonatal intensive care has long been recognised as an event that can substantially impact on parents. ${ }^{1}$ The questions addressed in this paper concern the extent to which the reactions differ from that of parents whose newborns are not admitted to a neonatal intensive care unit (NICU) and the impact on fathers as well as mothers.

Conventional models of becoming a parent contrast markedly with the NICU experience. With the latter, parental responses may be considered part of normal adjustment and transitory in nature, although if their infant is very sick and subsequently disabled, they may be part of a longer life changing process. $^{23}$ The study aimed to understand the nature of the impact of NICU on the wellbeing of both parents and to consider possible implications for practice and care. The objective could have been achieved in a number of ways and with different time frames. In this study the choice was made to assess psychosocial functioning at less than three weeks after the birth and admission to NICU using clinical interviews and self report questionnaires consisting of scales measuring symptoms of anxiety and depression, social adjustment, and the quality of the couple relationship. Using the same measures, direct comparisons were made with the responses of parents whose infants were not admitted to NICU and who were discharged home relatively soon after the birth.

The finding that a higher proportion of NICU parents suffered from anxiety, were more likely to have experienced a previous NICU admission, and had less financial resources to draw upon confirms what other studies have found. ${ }^{45}$ Similarly, the finding that, for NICU parents, having an infant who was born at a lower gestational age is associated with poorer mental health and wellbeing after their baby's birth supports the conclusions of other researchers. ${ }^{5}{ }^{6} \mathrm{How}$ ever, in the context of a relative paucity of data collected on NICU and non-NICU fathers, the study is particularly useful, although as the authors point out, slightly different scoring may have been preferable when using the depression symptoms scale with postnatal fathers.

The overall levels of anxiety and depression reported for mothers and fathers were relatively low in both groups of parents in comparison with those described in other studies, ${ }^{78}$ although the figures presented are within the prevalence range described in a meta-analysis on the measurement of depression postnatally. ${ }^{9}$ More significantly, there are methodological difficulties in making comparisons between studies on this topic: these include the employment of similar, but slightly different, instruments, the selected use of subscales, differences in the scoring cut off chosen, variation in the inclusion of possible moderating and confounding variables in the analyses, whether point or period prevalence is calculated, and differences in timing of administration from three days to 12 months or more after birth. The last point is critical in relation to this study in which data were collected on one occasion very early in the postpartum period. For women following the birth of a normal full term infant, depression has been described as peaking four to six weeks after the birth, hence the development of screening around this time. $^{10}$ However, the time course for symptoms of anxiety and depression consequent on the birth of a normal baby are not necessarily the same; they may not be the same for mothers and fathers, nor can they be assumed to be the same for parents whose baby is born preterm or with a medical condition necessitating admission to NICU. Thus in studies of this group of NICU parents and any comparison group, some authors have emphasised the importance of assessing parental mental health and psychosocial functioning more than once and the value of longer term follow up where possible. $^{371112}$ Bearing in mind the "roller coaster" of emotional peaks and troughs that many NICU parents describe as their experience of neonatal care and the transition to home, it seems that their "response" would be best assessed at a number of time points.

Parental exposure to NICU and the kind of care their infant experienced gestational ages. However, severity of illness and the duration of time that infants received intensive care were not included in the study variables analysed in relation to parental wellbeing. To conclude at this stage, without some longer term follow up of the participants, as the authors do, that most NICU parents make a successful adaptation and require little in the way of specific interventions may be premature. Baseline care in terms of the provision of a "parent friendly" environment varies considerably between units and may indeed in some centres obviate the need for intervention with most families. However, the link described between infant prematurity and poorer parental wellbeing does support the identification of a need for more targeted interventions. That such interventions should include fathers is clearly indicated by the findings presented, which effectively highlight their needs at this time as well as those of the partners. Arch Dis Child Fetal Neonatal Ed 2005;90:F96. doi: 10.1136/adc.2004.055426

Correspondence to: Dr Redshaw, National Perinatal Epidemiology Unit, University of Oxford, Old Road Campus, Headington Oxford OX3 7LF, UK; maggie.redshaw@ perinat.ox.ac.uk

Competing interests: none declared

\section{REFERENCES}

1 Klaus MH, Kennell JH. Maternal-infant bonding. St Louis: CV Mosby Co, 1976

2 Miles MS, Holditch-Davis D, Burchinal P, et al. Distress and growth outcomes in mothers of medically fragile infants. Nurs Res 1999:48:129-40.

3 Singer LT, Salvator A, Guo S, et al. Maternal psychological distress and parenting stress after the birth of a very low-birth-weight infant. JAMA 1999;281:799-805.

4 Cronin CM, Shapiro CR, Casiro OG, et al. The impact of very low-birth-weight infants on the family is long lasting. A matched control study. Arch Pediatr Adolesc Med 1995;149:151-8.

5 Meyer EC, Garcia Coll CT, Seifer R, et al. Psychological distress in mothers of preterm infants. J Dev Behav Pediatr 1995;16:412-17.

6 Holditch-Davis D, Bartlett TR, Blickman AL, et al. Posttraumatic stress symptoms in mothers of premature infants. J Obstet Gynecol Neonatal Nurs 2003;32:161-71.

7 Areias M, Kumar R, Barras $\mathrm{H}$, et al. Correlates of postnatal depression in mothers and fathers. Br J Psychiatry 1996:36-41.

8 Elliott SA, Leverton TJ. Is the EPDS a magic wand? 2. 'Myths' and the evidence base. J Reprod Infant Psychol 2000;18:297-307.

9 O'Hara MW, Swain AM. Rates and risk of postnatal depression: a meta-analysis. Int $\operatorname{Rev}$ Psychiatry 1996;8:37-54

10 Leverton TJ, Elliott SA. Is the EPDS a magic wand? 1. A comparison of the Edinburgh Postnatal Depression Scale and health visitor report as predictors of diagnosis on the Present State Examination. J Reprod Infant Psychol 2000; 18:279-96.

11 Miles MS, Holditch-Davis D, Burchinal P, et al. Distress and growth outcomes in mothers of medically fragile infants. Nurs Res 1999:48:129-40.

12 Pierrehumbert B, Nicole A, Muller-Nix C, et al. Parental post-traumatic reactions after premature birth: implications for sleeping and eating problems in the infant. Arch Dis Child Feta Neonatal Ed 2003;88:F400-4. 